\title{
Brain Emotional Learning Based Intelligent Decoupler for Nonlinear Multi-Input Multi-Output Distillation Columns
}

\author{
M. H. El-Saify, ${ }^{1}$ A. M. El-Garhy, ${ }^{1}$ and G. A. El-Sheikh ${ }^{2}$ \\ ${ }^{1}$ Electronics, Communications, and Computers Department, Faculty of Engineering, Helwan University, Cairo, Egypt \\ ${ }^{2}$ Electronics and Communications Department, Pyramids High Institute (PHI) for Engineering and Technology, \\ 6th of October, Giza, Egypt
}

Correspondence should be addressed to M. H. El-Saify; mhelsaify@hotmail.com

Received 27 August 2016; Revised 24 December 2016; Accepted 9 January 2017; Published 31 January 2017

Academic Editor: Asier Ibeas

Copyright (C) 2017 M. H. El-Saify et al. This is an open access article distributed under the Creative Commons Attribution License, which permits unrestricted use, distribution, and reproduction in any medium, provided the original work is properly cited.

\begin{abstract}
The distillation process is vital in many fields of chemical industries, such as the two-coupled distillation columns that are usually highly nonlinear Multi-Input Multi-Output (MIMO) coupled processes. The control of MIMO process is usually implemented via a decentralized approach using a set of Single-Input Single-Output (SISO) loop controllers. Decoupling the MIMO process into group of single loops requires proper input-output pairing and development of decoupling compensator unit. This paper proposes a novel intelligent decoupling approach for MIMO processes based on new MIMO brain emotional learning architecture. A MIMO architecture of Brain Emotional Learning Based Intelligent Controller (BELBIC) is developed and applied as a decoupler for 4 input/4 output highly nonlinear coupled distillation columns process. Moreover, the performance of the proposed Brain Emotional Learning Based Intelligent Decoupler (BELBID) is enhanced using Particle Swarm Optimization (PSO) technique. The performance is compared with the PSO optimized steady state decoupling compensation matrix. Mathematical models of the distillation columns and the decouplers are built and tested in simulation environment by applying the same inputs. The results prove remarkable success of the BELBID in minimizing the loops interactions without degrading the output that every input has been paired with.
\end{abstract}

\section{Introduction}

In chemical industries, distillation is one of the most important processes. The objective of distillation is to separate a mixture of chemical components. The purpose of control is to maintain bottom and top product (distillate) purity despite variations in feed flow and feed concentration. As the distillation columns process is a MIMO system, many researches utilize decentralized approaches to control it [1]. The two-coupled distillation columns is a 4 input/ 4 output process. Normally, control engineers decouple the process into four independent loops via a decoupler [2]. Then, every loop is controlled separately by SISO controller [3]. This work is aimed at proposing MIMO decoupling unit based on brain emotional learning technique and applying it to the twocoupled distillation columns.

The design of intelligent systems is one of the most growing fields that have received considerable attentions in recent years. Biologically motivated intelligent control is the discipline in which control algorithms are developed by emulating certain characteristics of intelligent biological systems. Many control techniques, such as artificial neural networks $[4,5]$, fuzzy control $[6,7]$, and genetic algorithms [8,9], had proven its effectiveness in solving wide range of complex control problems. Recently, a new member was added to this family of biologically motivated intelligent control, which mimics the emotional learning process in the limbic system of the mammalian brains. The model of brain emotional learning algorithm had been proposed in $[10,11]$ and then was developed and shared for control engineering applications [12]. Since then, BELBIC is increasingly being utilized in many control engineering applications such as electric motors [13, 14], servo systems $[15,16]$, motion control $[17,18]$, and power systems $[19,20]$. Some recent researches utilize other intelligent techniques in cooperation with BELBIC to control the system such as fuzzy logic $[21,22]$. These applications had utilized BELBIC as a SISO system. In [3], the two-coupled distillation columns is 
controlled by 4 separate BELBIC controllers and a steadystate decoupling compensation unit. BELBIC controllers had proven its effectiveness in controlling the distillation columns compared with the particle swarm optimized PID controllers. This paper proposes a new MIMO structure of the BELBIC model which is utilized as a decoupler of MIMO system.

PSO, introduced by Kennedy and Eberhart [23] and Eberhart et al. [24], is a population-based stochastic approach for solving continuous and discrete optimization problems. In PSO, simple software agents, called particles, move in the search space of an optimization problem. The position of a particle represents a candidate solution to the optimization problem at hand. Each particle searches for better positions in the search space by changing its velocity according to rules originally inspired by behavioral models of bird or fish flocking. In this paper, PSO is used to optimize the performance of the BELBID.

The rest of this paper is organized as follows: A literature review including mathematical modeling of the two-coupled distillation columns is presented in Section 2. In Section 3, a literature review including mathematical modeling of brain emotional learning and its applications is presented. In Section 4, a new MIMO brain emotional learning model is introduced and utilized to work as a decoupler for the twocoupled distillation columns. In Section 5, the simulation and results are discussed. Finally, the paper is concluded with brief remarks in Section 6.

\section{Modeling and Decoupling of the Two-Coupled Distillation Columns Process}

2.1. Mathematical Modeling of the Two-Coupled Distillation Columns. The distillation process is an active topic in chemical and control research fields. Modeling and control of the distillation process is vital for the chemical industry. Many researches are conducted to model the distillation process dynamics and to provide a robust control of it [25-28]. Among the distillation processes, the two-coupled distillation columns is a 4 input/4 output process as shown in Figure 1. The objective of distillation process is to separate a mixture of chemical components based on the differences of the boiling points of these components. The outputs are tray temperatures: $T_{11}, T_{30}, T_{34}$, and $T_{48}$. Keeping the tray temperatures within a specified range around their steady state values is essential for specifying top and bottom product purity. The inputs are the heat added (QE), steam from column $A$ to column B (SAB), reflux produced from column A (RLA), and reflux produced from column $B(R L B)$. More details are listed in $[2,3]$. The two-coupled distillation columns process is described by the following transfer function equations:

$$
\begin{aligned}
{\left[\begin{array}{l}
T_{11} \\
T_{30} \\
T_{34} \\
T_{48}
\end{array}\right] } & =H(s)\left[\begin{array}{c}
\mathrm{QE} \\
\mathrm{SAB} \\
\mathrm{RLA} \\
\mathrm{RLB}
\end{array}\right], \\
H(s) & =\left[\begin{array}{cccc}
\frac{2.6}{1.96 s+1} & \frac{-6.098}{3.5 s+1} & \frac{-4.99(0.2 s+1)}{(4.5 s+1)(0.06 s+1)} & \frac{0.071}{3.5 s+1} \\
\frac{7.32(1.05 s+1)}{(10.4 s+1)(0.14 s+1)} & \frac{-1.45}{0.4 s+1} & \frac{-1.57(0.23 s+1)}{(1.34 s+1)(0.2 s+1)} & \frac{-0.14}{1.92 s+1} \\
\frac{4.6(0.53 s+1)}{(2.78 s+1)(0.09 s+1)} & \frac{-2.37(0.23 s+1)}{(2 s+1)(0.3 s+1)} & \frac{-2.7}{1.75 s+1} & \frac{-0.36(0.02 s+1)}{(2.47 s+1)(0.04 s+1)} \\
\frac{2.11}{0.92 s+1} & \frac{-2.11(0.06 s+1)}{(2.38 s+1)(0.05 s+1)} & \frac{-1.75}{2.16 s+1} & \frac{-0.3(1.89 s+1)}{(4.35 s+1)(0.16 s+1)}
\end{array}\right] .
\end{aligned}
$$

2.2. Decoupling of the Two-Coupled Distillation Columns. Two main approaches are utilized to control MIMO systems, namely, centralized and decentralized techniques. Papers that have addressed the decentralized control deal with resolution of two design problems, namely, proper input-output pairing and interaction minimization. The proper input-output pairing is usually achieved through the development of relative gain array (RGA) $[29,30]$. The interaction minimization is achieved through the design of decoupling compensator matrix, which was introduced in many papers $[31,32]$. The design of decoupling unit includes estimating the elements' values of steady state decoupling compensation matrix using detailed analytical techniques with very high mathematical burdens, specially, for high order MIMO processes. After decoupling, a set of SISO controllers are used to control the system in a decentralized way. Figure 2 shows general 2 input/2 output decoupled control system. In Figure 2, G represent SISO controller, $\lambda$ represents compensation matrix parameter, and $H$ represents the coupled system transfer function. This technique was applied to the two-coupled distillation columns (4 input/4 output process); then the decoupling compensation matrix is optimized via PSO in [2]. This work will be considered for comparison and evaluation of our work.

The decentralized approach, which is using a set of SISO controllers to control the process, is preferred by many researchers $[33,34]$. Using a decoupling unit decreases the interactions between the controlled loops. The decoupling compensation matrix proposed in [2] is used in further research [3]. According to [2], the coupled pairs are chosen 


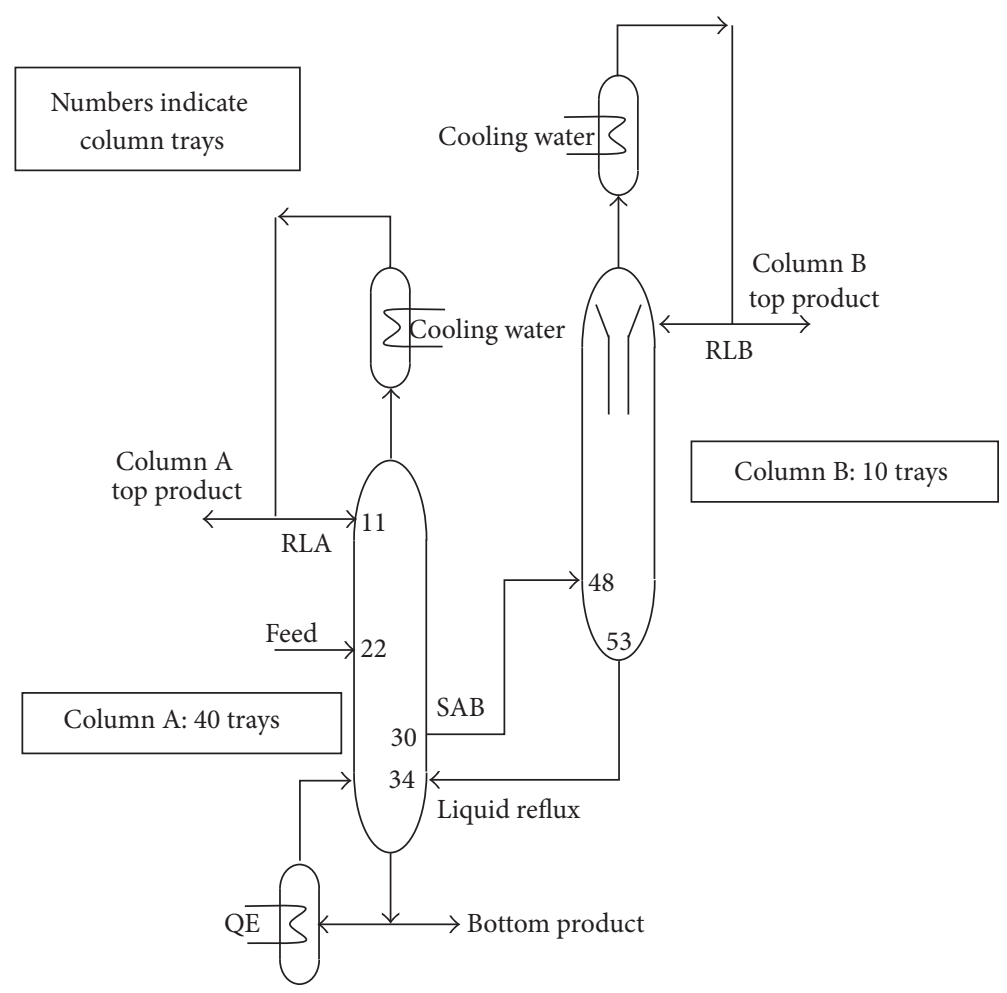

FIGURE 1: Graphical representation of the two-coupled distillation columns process.

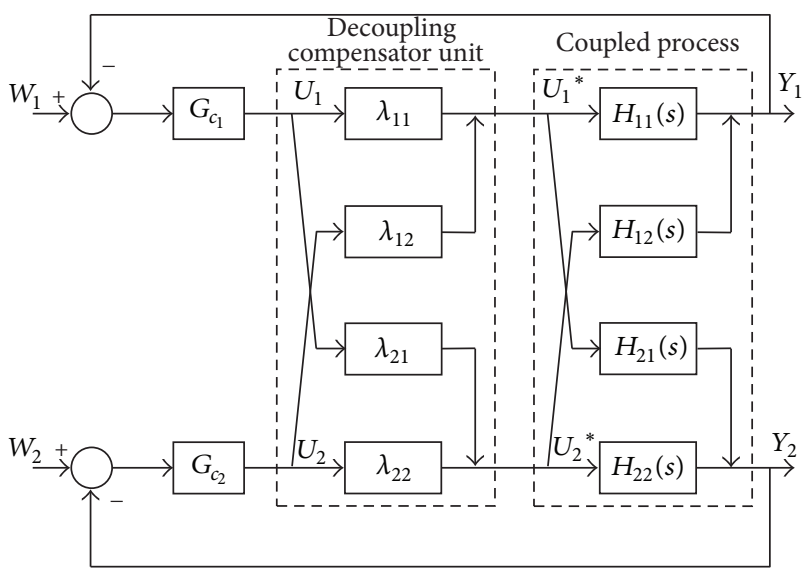

FIGURE 2: 2 input/2 output decentralized control system.

to be $\left(\mathrm{QE}, T_{30}\right),\left(\mathrm{SAB}, T_{11}\right),\left(\mathrm{RLA}, T_{34}\right)$, and $\left(\mathrm{RLB}, T_{48}\right)$. In addition, the steady state decoupling compensation matrix is given in the following equations and connected as shown in Figure 3:

$$
\left[\begin{array}{c}
T_{11} \\
T_{30} \\
T_{34} \\
T_{48}
\end{array}\right]=H \Lambda\left[\begin{array}{c}
\mathrm{QE} \\
\mathrm{SAB} \\
\mathrm{RLA} \\
\mathrm{RLB}
\end{array}\right]
$$

where $H$ is the process transfer function given in (2), and $\Lambda$ is the steady state decoupling compensation matrix given by [2]

$$
\Lambda=\left[\begin{array}{cccc}
1 & 0.1788 & 0.0608 & -0.0078 \\
-1.9273 & 1 & -0.7906 & 0.4555 \\
2.9263 & 0.8865 & 1 & -0.5466 \\
3.5183 & -10.9464 & 0.1548 & 1
\end{array}\right]
$$

Figure 3 declares the decoupled system.

\section{Brain Emotional Learning}

3.1. Computational Model of Emotional Learning. The brain emotional learning was introduced for the first time by Balkenius and Morén [10, 11]. They developed a computational model that mimics the parts of the mammalian brain that process the emotions. The model is powerful in real time control and decision making systems due to its simplicity, low computational complexity, and fast training [35]. The model is successfully utilized in many fields including system identification and prediction [36, 37], forecasting [38, 39], and pattern recognition [40].

Amygdala, orbitofrontal cortex, thalamus, and sensory input cortex are parts of the brain thought to be responsible for processing of emotions. The sensory input signals are received via the thalamus. After preprocessing in the thalamus, processed input signal will be sent to the amygdala and the sensory cortex. The amygdala and the orbitofrontal cortex are used to compute their outputs based on emotional 


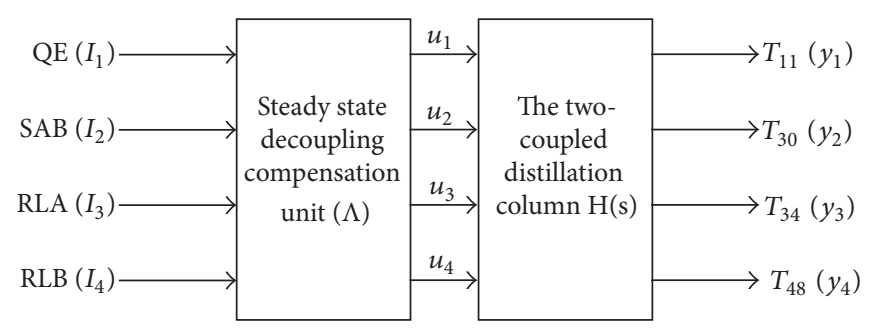

FIGURE 3: The decoupled two-coupled distillation columns process.

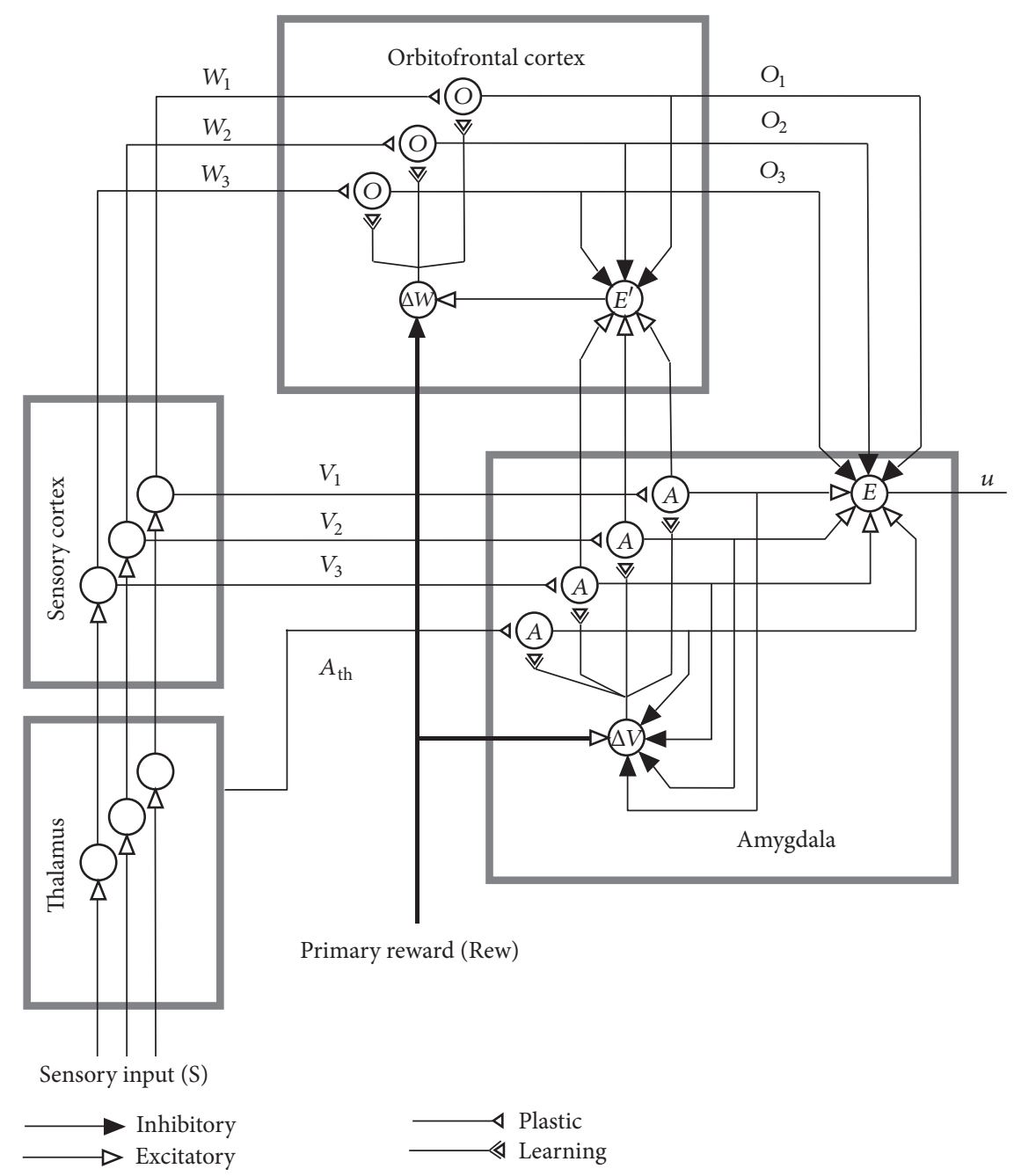

FIgURE 4: Graphical depiction of brain emotional learning.

signal (primary reward or reinforcing signal) received from the environment. The final output is calculated by subtracting the amygdala and the orbitofrontal cortex outputs. More details are declared in [10-12]. Figure 4 represents a graphical depiction of brain emotional learning.

As shown in Figure 4, the vector $S$ shows stimuli inputs to the system. There is one $A$ node for each stimulus $S . A_{\text {th }}$ is a node in the amygdala which directly receives the maximum stimuli signals via a path from the thalamus:

$$
A_{\text {th }}=\max \left(S_{i}\right) \text {. }
$$

The output of each node $A$ is calculated based on the multiplication of prespecified plastic connection weight $(V)$ into the corresponding input:

$$
A_{i}=S_{i} V_{i}
$$

The $V_{i}$ is adjusted proportionally to the difference between the activation of the $A$ nodes and the reinforcing signal Rew. The $\alpha$ term is a constant used to adjust the learning speed: 


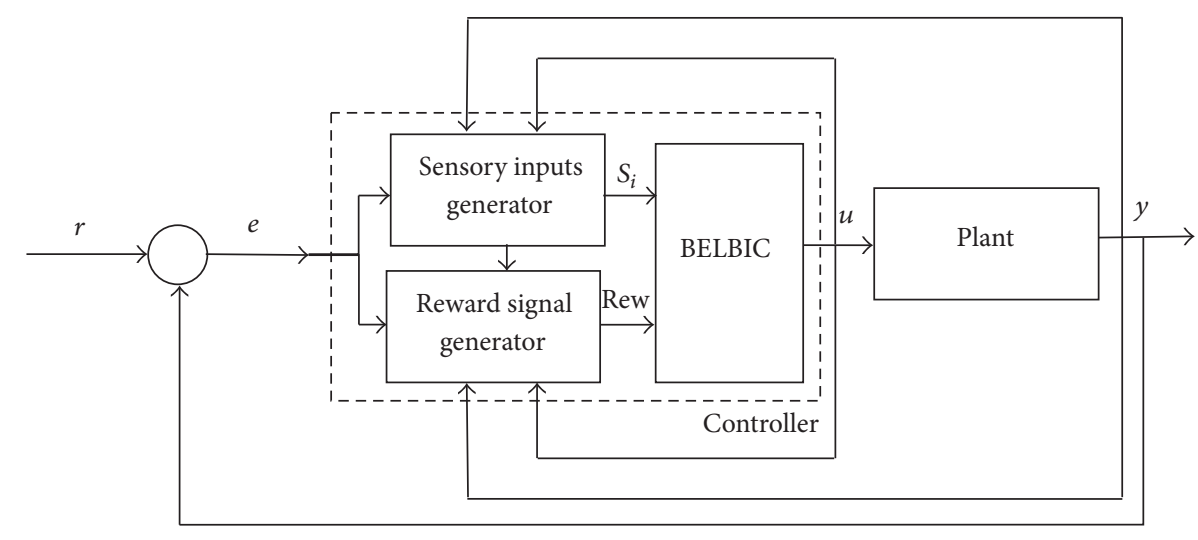

FIGURE 5: Control system configuration using BELBIC as a main controller.

$$
\Delta V_{i}=\alpha\left(S_{i} \max \left(0, \text { Rew }-\sum_{j} A_{j}\right)\right) .
$$

The weights $V$ cannot decrease, and it is a good reason for this design choice because once an emotional reaction is learned, this should be permanent and cannot be unlearned. It is the task of the orbitofrontal part to inhibit this reaction when it is inappropriate. The orbitofrontal learning rule is very similar to the amygdala rule but the orbitofrontal connection weight can both increase and decrease. The $O$ nodes behave analogously, with a connection weight $W$ employed to the input signal to create an output

$$
O_{i}=S_{i} W_{i}
$$

$\Delta W_{i}$ is calculated as

$$
\Delta W_{i}=\beta\left(S_{i}\left(E^{\prime}-\mathrm{Rew}\right)\right),
$$

where $\beta$ is another learning rate constant. The $E$ node simply sums the outputs from the $A$ nodes and then subtracts the inhibitory outputs from the $O$ nodes. The result is the output from the model. The $A$ nodes give outputs proportionally to their contribution in predicting the reward Rew, while the $O$ nodes inhibit the output of $E$ as necessary. The $E^{\prime}$ node sums the outputs from $A$ except $A_{\text {th }}$ and then subtracts from inhibitory outputs from the $O$ nodes:

$$
\begin{aligned}
E & =\sum_{i} A_{i}-\sum_{i} O_{i} \quad\left(\text { including } A_{\mathrm{th}}\right) \\
E^{\prime} & =\sum_{i} A_{i}-\sum_{i} O_{i} \quad\left(\text { not including } A_{\mathrm{th}}\right) .
\end{aligned}
$$

3.2. BELBIC Model. There are two main approaches of applying the brain emotional learning in a control process: either using BELBIC as a controller or using it to adapt an existing controller online. Figure 5 declares BELBIC as a main controller within a typical feedback control block diagram.

The choice of sensory inputs and reward signal differs from research to another. Generally, the sensory inputs must include signals that provide necessary information to control the system (e.g., the plant output, the controller output, the error, etc.). These signals are chosen and reshaped in a function chosen by BELBIC designer to produce the sensory inputs. On the other hand, the reward signal comes as a function of signals which can validate a cost function chosen by the designer. The output is enforced by the reward signal (by applying reward or punishment) to validate the preselected cost function. Sensory inputs and reward signal are represented mathematically as follows:

$$
\begin{aligned}
S_{i} & =f_{i}(e, u, y), \\
\text { Rew } & =J\left(S_{i}, e, u, y\right),
\end{aligned}
$$

where $e$ is the error between plant output $y$ and reference signal $r$, while $u$ is the BELBIC output. Each research dedicates part of its effort to develop sensory input generator and reward signal generator. In addition, many researches optimize the gains of the generators by an optimization technique. Among other techniques, particle swarm optimization is utilized for optimizing the gains of the generators and the learning rates of the BELBIC [3].

Particle Swarm Optimization. Particle swarm optimization is a heuristic global optimization method. It is based on the research of bird and fish flock movement behavior. In PSO algorithm, particle swarm consists of $M$ particles $(i=1,2, \ldots, M)$, and the position of each particle $x_{i d}$ represents the potential solution in $D$-dimensional space $\left(x_{i 1}, x_{i 2}, \ldots, x_{i d}, \ldots, x_{i D}\right)$. The particles change its condition (velocity $\left(\nu_{i d}\right)$ and position $\left(x_{i d}\right)$ ) according to its most optimist position $\left(p_{i d}\right)$ and the swarm's most optimist position $\left(p_{g d}\right)$ while maintaining its inertia by a preselected factor called inertia weight $\left(w_{i}\right)$. The following equations represent the particles movement in the search space:

$$
\begin{aligned}
& v_{i d}^{z+1}=w_{i}^{z+1} v_{i d}^{z}+c_{1} r_{1}^{z}\left(p_{i d}^{z}-x_{i d}^{z}\right)+c_{2} r_{2}^{z}\left(p_{g d}^{z}-x_{i d}^{z}\right), \\
& x_{i d}^{z+1}=x_{i d}^{z}+v_{i d}^{z+1},
\end{aligned}
$$

where $z=1,2, \ldots, n_{z}$ determines the iteration number and $n_{z}$ is the maximal times of iteration. There are many approaches to choose $w_{i}$, which is used to adjust the global and local 
searching capability. One of these approaches is decreasing $w_{i}$ gradually (from 0.9 to 0.4 ) during the iteration.

\section{Proposed MIMO Brain Emotional Learning}

4.1. Proposed MIMO-BELBIC Model. The BELBIC model introduced by [12] has one output. It is used widely to control SISO linear and nonlinear systems. The BELBIC controllers have the capability to overcome uncertainty and complexity issues of control applications. Although it is used to control MIMO systems in some applications [12, 41], the researchers used a set of BELBIC controllers to control every loop/output individually. In other words, they used the single output BELBIC model introduced in [12] rather than MIMO model. In $[12,41]$, they used 2 and 4 BELBIC controllers, respectively. In this research, we introduce MIMO-BELBIC model that can be utilized to control many loops/outputs simultaneously. This configuration allows the model to control every loop taking into consideration the state of other loops. This property allows compensating for the interactions between loops and providing more stable performance. Therefore, as a first application of the MIMO-BELBIC model, we applied the model as a decoupler to test its ability to compensate for the interactions between the controlled loops.

Many emotions can be generated in the mammalian brains. Sometimes, the same action (sensory inputs) can generate more than one emotion simultaneously. Receiving a gift may make us feel happy, hopeful, grateful, and so forth. The paper proposes a new brain emotional learning model, which is MIMO, (MIMO-BELBIC model) as illustrated in Figure 6. As declared in Figure 6, the new brain emotional learning model suggests that every emotion (output) has its $A, O, E$, and $E^{\prime}$ nodes and is connected to all necessary sensory inputs. Each emotion has its reinforcing signal which validates its cost function and its own sensory inputs if necessary. Sensory inputs generator and reward signals generator are involved to build MIMO-BELBIC model. All the gains of the MIMO-BELBIC in addition to learning rates should be optimized together to minimize a specified cost function.

The following sets of equations are considered for the MIMO-BELBIC:

$A_{1 \mathrm{th}}=\max \left(S_{1 i}\right)$,

$A_{N \text { th }}=\max \left(S_{N i}\right)$,

$A_{1 i}=S_{1 i} V_{1 i}$

$A_{N i}=S_{N i} V_{N i}$

$\Delta V_{1 i}=\alpha_{1}\left(S_{1 i} \max \left(0, \operatorname{Rew}_{1}-\sum_{j} A_{1 j}\right)\right)$,
$\Delta V_{N i}=\alpha_{N}\left(S_{N i} \max \left(0, \operatorname{Rew}_{N}-\sum_{j} A_{N j}\right)\right)$,

$O_{1 i}=S_{1 i} W_{1 i}$

$O_{N i}=S_{N i} W_{N i}$

$\Delta W_{1 i}=\beta_{1}\left(S_{1 i}\left(E_{1}^{\prime}-\operatorname{Rew}_{1}\right)\right)$,

$\Delta W_{N i}=\beta_{N}\left(S_{N i}\left(E_{N}^{\prime}-\operatorname{Rew}_{N}\right)\right)$,

$E_{1}=\sum_{i} A_{1 i}-\sum_{i} O_{1 i} \quad\left(\right.$ including $\left.A_{1 \mathrm{th}}\right)$

$E_{N}=\sum_{i} A_{N i}-\sum_{i} O_{N i} \quad\left(\right.$ including $\left.A_{N \mathrm{th}}\right)$,

$E_{1}^{\prime}=\sum_{i} A_{1 i}-\sum_{i} O_{1 i} \quad\left(\right.$ not including $\left.A_{\mathrm{th}}\right)$,

$E_{N}^{\prime}=\sum_{i} A_{N i}-\sum_{i} O_{N i} \quad\left(\right.$ not including $\left.A_{\mathrm{th}}\right)$,

$S_{i 1}=f_{i 1}\left(e_{1}, \ldots, e_{N}, u_{1}, \ldots, u_{N}, y_{1}, \ldots, y_{N}\right)$,

$S_{i N}=f_{i N}\left(e_{1}, \ldots, e_{N}, u_{1}, \ldots, u_{N}, y_{1}, \ldots, y_{N}\right)$,

$\operatorname{Rew}_{1}=J_{1}\left(S_{1 i}, \ldots, S_{N i}, e_{1}, \ldots, e_{N}, u_{1}, \ldots, u_{N}, y_{1}, \ldots, y_{N}\right)$,

$\operatorname{Rew}_{N}=J_{N}\left(S_{1 i}, \ldots, S_{N i}, e_{1}, \ldots, e_{N}, u_{1}, \ldots, u_{N}, y_{1}, \ldots, y_{N}\right)$.

It is obvious that the reward signals are function of all inputs. This construction gives the ability to compensate for the coupling in order to decrease the loops interaction. The proposed MIMO-BELBIC is tailored in this paper to work as a decoupler of MIMO nonlinear systems, namely, BELBID. This approach is applied to the two-coupled distillation process and PSO is used to optimize the decoupler performance.

4.2. The Proposed BELBID. The proposed BELBID model is 4 input/4 output BELBIC and is employed as a decoupler for 


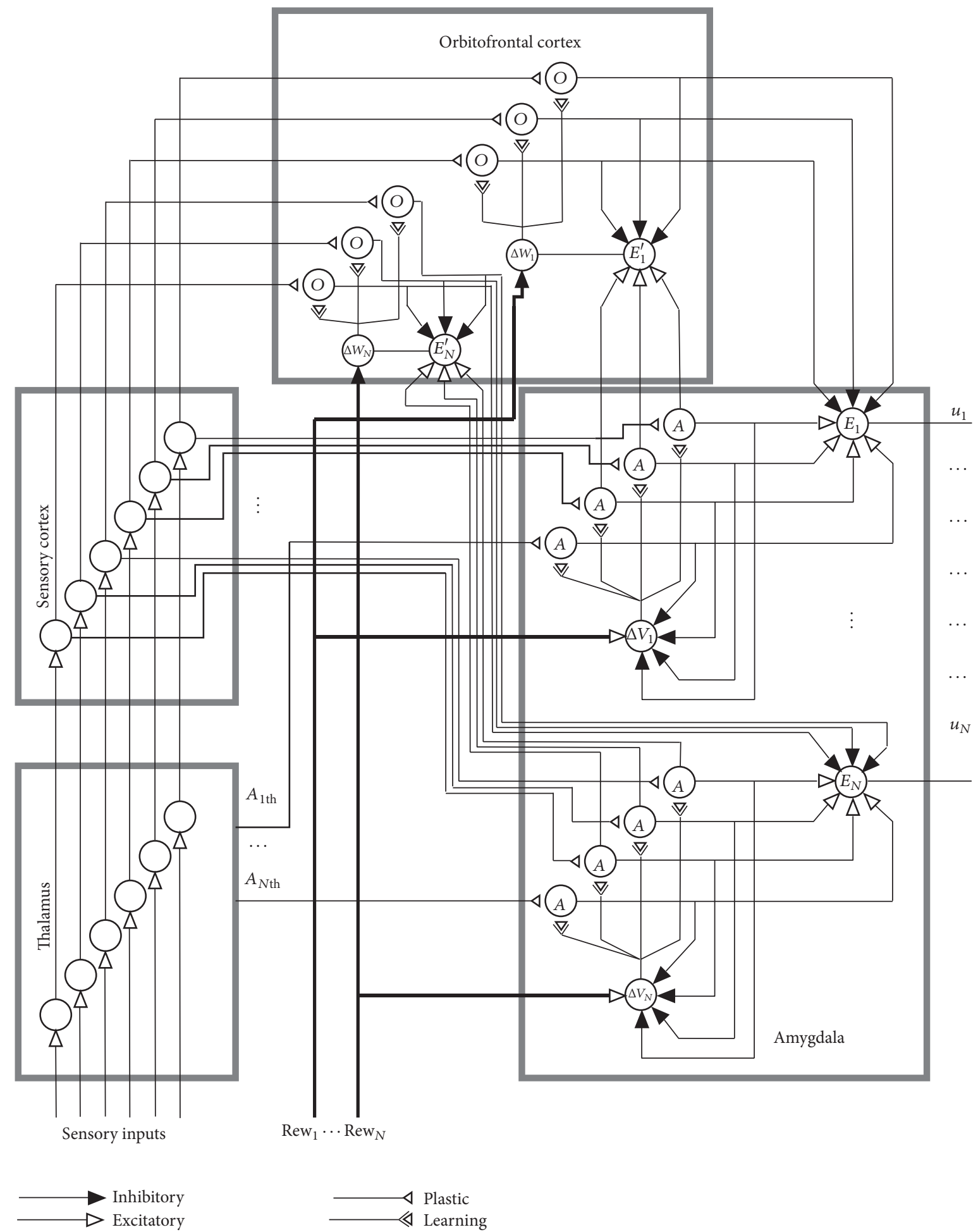

FIgURE 6: Graphical depiction of MIMO brain emotional learning.

the two-coupled distillation columns process. We produce 16 sensory input and 4 reward signals. The sensory signals are divided into 4 groups, 4 signals each. Each group of sensory signal is fed to $A$ nodes and $O$ nodes belong to one decoupler output as shown in Figure 7. In this design, $A_{\text {th }}$ is set to zero (i.e., there is no maximum stimuli signal output from the thalamus). The following equations represent the first group of sensory inputs which are related to the BELBID output $u_{1}$ :

$$
\begin{aligned}
& s_{1}=k_{p 11} I_{1}+k_{i 11} \int I_{1} d t, \\
& s_{2}=k_{p 12} I_{2}+k_{i 12} \int I_{2} d t,
\end{aligned}
$$




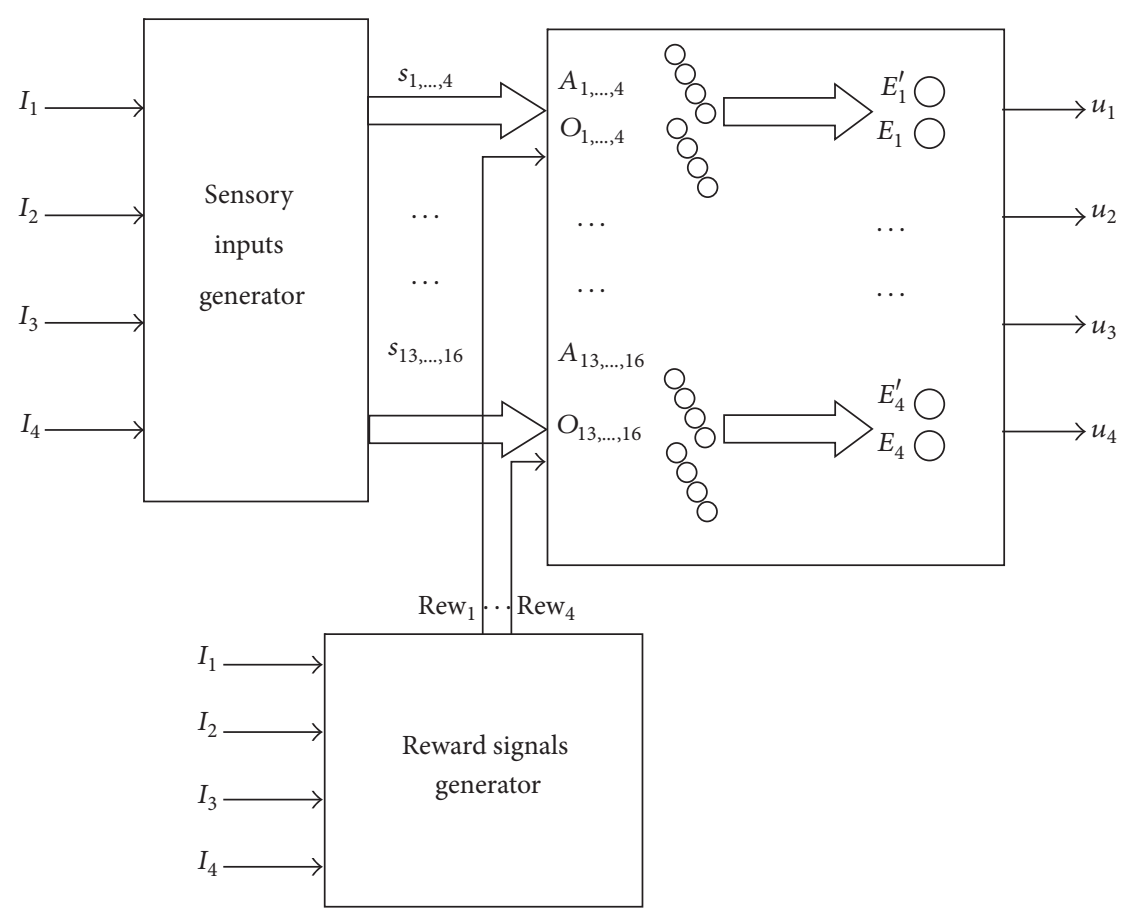

FIGURE 7: Internal connections of 4 input/4 output BELBID.

$$
\begin{aligned}
& s_{3}=k_{p 13} I_{3}+k_{i 13} \int I_{3} d t, \\
& s_{4}=k_{p 14} I_{4}+k_{i 14} \int I_{4} d t .
\end{aligned}
$$

The $k_{p 11}, k_{p 12}, \ldots$, and so forth are gains to be optimized. The following equation represents the first reward signal:

$$
\begin{aligned}
\operatorname{Rew}_{1}= & k_{r p 11} I_{1}+k_{r d 11} \frac{d}{d t} I_{1}+k_{r i 11} \int I_{1} d t+k_{r p 12} I_{2} \\
& +k_{r d 12} \frac{d}{d t} I_{2}+k_{r i 12} \int I_{2} d t+k_{r p 13} I_{3} \\
& +k_{r d 13} \frac{d}{d t} I_{3}+k_{r i 13} \int I_{3} d t+k_{r p 14} I_{4} \\
& +k_{r d 14} \frac{d}{d t} I_{4}+k_{r i 14} \int I_{4} d t .
\end{aligned}
$$

The rest of sensory inputs and reward signals equations are defined by the analogy to (14)-(15). Note that the above constants $\left(k_{p 11}, k_{i 11}, k_{p 12}, k_{i 12}\right.$, etc. $)$ are related to the BELBID output $u_{1}$ and it will be called $\left(k_{p 21}, k_{i 21}, k_{p 22}, k_{i 22}\right.$, etc. ) for $u_{2}$ and so on.

All gains in addition to learning rate constants $\left(\alpha_{1,2,3,4}\right.$, $\left.\beta_{1,2,3,4}\right)$ are optimized via PSO. The inertia weight $w_{i}$ of the PSO is reduced gradually from 0.9 to 0.4 . Step changes are applied at the inputs, one at a time, and zero are applied to other inputs. For ideal decoupler, all outputs of the process should remain zero except the output paired to this input.
So, the responses at the unpaired outputs are considered as a coupling error. The best solution is chosen to be the minimum total coupling error. For PSO, number of particles is chosen to be 100 and both $c_{1}$ and $c_{2}$ are set to 0.5 . The cost function which is used to evaluate the particles and to be minimized is the sum of all coupling errors of all unpaired outputs. The errors are represented by the Integral Squared Error (ISE) form. Regarding that the paired inputs and outputs are $\left(I_{1}-y_{2}\right.$, $\left.I_{2}-y_{1}, I_{3}-y_{3}, I_{4}-y_{4}\right)$, so the cost function $F_{m}$ is given by

$$
\begin{aligned}
F_{m}= & \int e_{11}^{2} d t+\int e_{13}^{2} d t+\int e_{14}^{2} d t+\int e_{22}^{2} d t+\int e_{23}^{2} d t \\
& +\int e_{24}^{2} d t+\int e_{31}^{2} d t+\int e_{32}^{2} d t+\int e_{34}^{2} d t \\
& +\int e_{41}^{2} d t+\int e_{42}^{2} d t+\int e_{43}^{2} d t
\end{aligned}
$$

where $e_{i j}$ is the coupling error at output $j$ due to input $i$. Generally, the cost function can be written as follows:

$$
F_{m}=\sum_{i=1}^{N} \sum_{j=1}^{N-1} \int e_{i j}^{2} d t,
$$

where $i$ and $j$ are unpaired inputs and outputs, respectively, and $N$ is the number of inputs/outputs.

The optimization results are detailed in Table 1.

\section{Simulation and Results}

The model is built in MATLAB environment, where the results are compared with the decoupling compensation 
TABLE 1: The optimized gains and learning rate constants of the BELBID.

(a)

\begin{tabular}{cccccccc}
\hline & & \multicolumn{3}{c}{ Sensory inputs } \\
& ${ }^{*} k_{p 11}$ & $k_{i 11}$ & $k_{p 12}$ & $k_{i 12}$ & $k_{p 13}$ & $k_{i 13}$ & $k_{p 14}$ \\
\hline$u_{1}$ & 0.91442 & 0.30504 & 0.98729 & $1.49 * 10^{-6}$ & 0.87959 & 0.02545 & 1.00007 \\
$u_{2}$ & 0.99802 & $-4.78 * 10^{-6}$ & 1.23042 & 1.68921 & 0.11573 & 0.76184 & 3.30517 \\
$u_{3}$ & 0.98515 & $1.21 * 10^{-4}$ & 0.76503 & -1.71535 & 0.93271 & $-1.09 * 10^{-4}$ & 5.19011 \\
$u_{4}$ & 1.07348 & -0.36335 & 0.59586 & 0.21164 & -0.3116 & -0.01136 & 1.003 \\
\hline
\end{tabular}

(b)

\begin{tabular}{cccccccc}
\hline & & \multicolumn{3}{c}{ Reward signals } & & $k_{r i 12}$ & $k_{r p 13}$ \\
\hline$u_{1}$ & $k_{r p 11}$ & $k_{r d 11}$ & $k_{r i 11}$ & $k_{r p 12}$ & $k_{r d 12}$ & 0.0608 \\
$u_{2}$ & 0.99997 & 14.12 & $-1.89 * 10^{-8}$ & 0.17881 & 45.74 & $-1.09 * 10^{-5}$ \\
$u_{3}$ & -1.9273 & 1.52 & $-5.74 * 10^{-8}$ & 0.99942 & 12.87 & $-1.93 * 10^{-4}$ & -0.7905 \\
$u_{4}$ & 2.92619 & 3.56 & $-4.52 * 10^{-6}$ & 0.8862 & 48.25 & $7.29 * 10^{-5}$ & 1.00002 \\
\hline
\end{tabular}

(c)

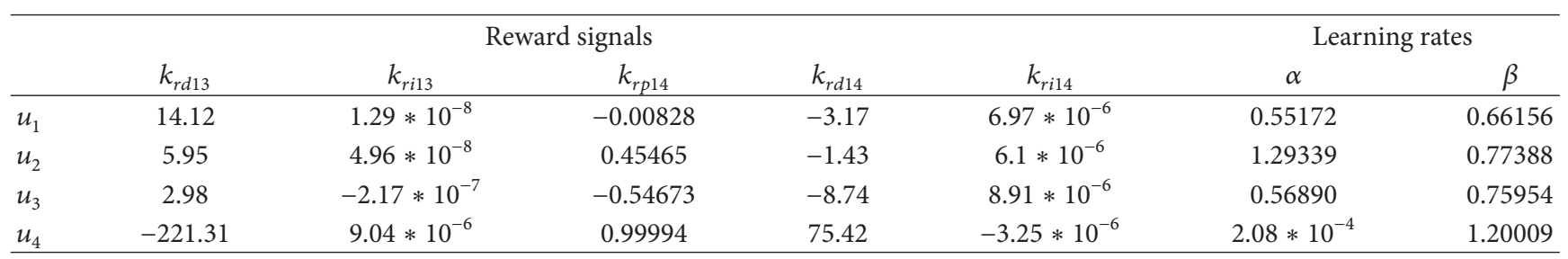

${ }^{*}$ Note that $k_{p 11}$ is for $u_{1}$, and it is called $k_{p 21}$ for $u_{2}$ and $k_{p 31}$ for $u_{3}$ and so on for all constants.

matrix provided in [2]. The authors of [2] proposed technique uses RGA to select proper pairing and PSO technique to estimate the optimal values of steady state decoupling compensation matrix constituting the decoupling compensator unit. The matrix is used in further works [3]. The model of the compensator matrix decoupler is built and compared with the BELBIC decoupler. Both models of the decouplers are tested by applying the same step changes in system inputs and the coupling errors for all unpaired outputs are measured by the technique descried in the last section. The sum of all integral squared errors due to coupling given by (17) is used to evaluate the decouplers. Equation (17) can be rewritten as follows:

$$
\mathrm{ISE}_{\text {total }}=\sum_{i=1}^{N} \sum_{j=1}^{N-1} \int e_{i j}^{2} d t=\sum_{i=1}^{N} \sum_{j=1}^{N-1} \mathrm{ISE}_{i j},
$$

where $i$ and $j$ represent the response of unpaired output $j$ due to input $i$. It is found that the BELBID remarkably decreases the total coupling error compared with the compensation matrix. The optimization process of the BELBID tunes its parameters simultaneously. As the cost function depends on the total error, it is expected that the best improvement of the BELBID should be in the worst case of the coupling. The detailed responses to the 4 inputs are declared in Figure 8. It is clear that the worst coupling error of the decoupled system by the compensation matrix occurs between input 1 and output 1. The ISE due to coupling between input 1 and output 1 exceeds $57 \%$ of total ISE. BELBID is remarkably decreases this coupling error. On the other hand, the BELBID does not degrade the outputs of the coupled pairs $\left(I_{1}-y_{2}, I_{2}-y_{1}\right.$, $I_{3}-y_{3}$, and $\left.I_{4}-y_{4}\right)$. Figure 9 compares the coupling errors of the two decouplers by a bar graph. It declares that the choice of the cost function as the sum of all coupling errors lets the optimization process give a higher priority to the biggest coupling errors. As shown in Figure 9, the biggest coupling errors of the compensation matrix are $e_{11}, e_{22}$, and $e_{31}$, respectively. These errors represent the main deficiency of the compensation matrix. It is clear that the BELBID overcomes this problem and remarkably decreases these errors. The integration in the ISE motivates the optimization process to prefer the solutions that produce coupling responses like narrow glitches that decay rapidly. All the 12 th coupling errors produced by the BELBID are smaller than the errors that are produced by the compensation matrix except $e_{14}$ and $e_{34}$. It is found that improving these errors will produce increase in more important errors and degrade the overall improvement. For example, improving $e_{14}$ will cause big increase in $e_{11}$ which is the most important error. Finally, according to (18), the total ISE due to coupling of the systems is reduced by $85.3 \%$ compared with the compensation matrix results.

\section{Conclusion}

In this paper, the control of distillation columns, which is usually highly nonlinear and coupled process, is enhanced by applying a novel MIMO model of brain emotional learning. The model mimics the brain ability to introduce many 

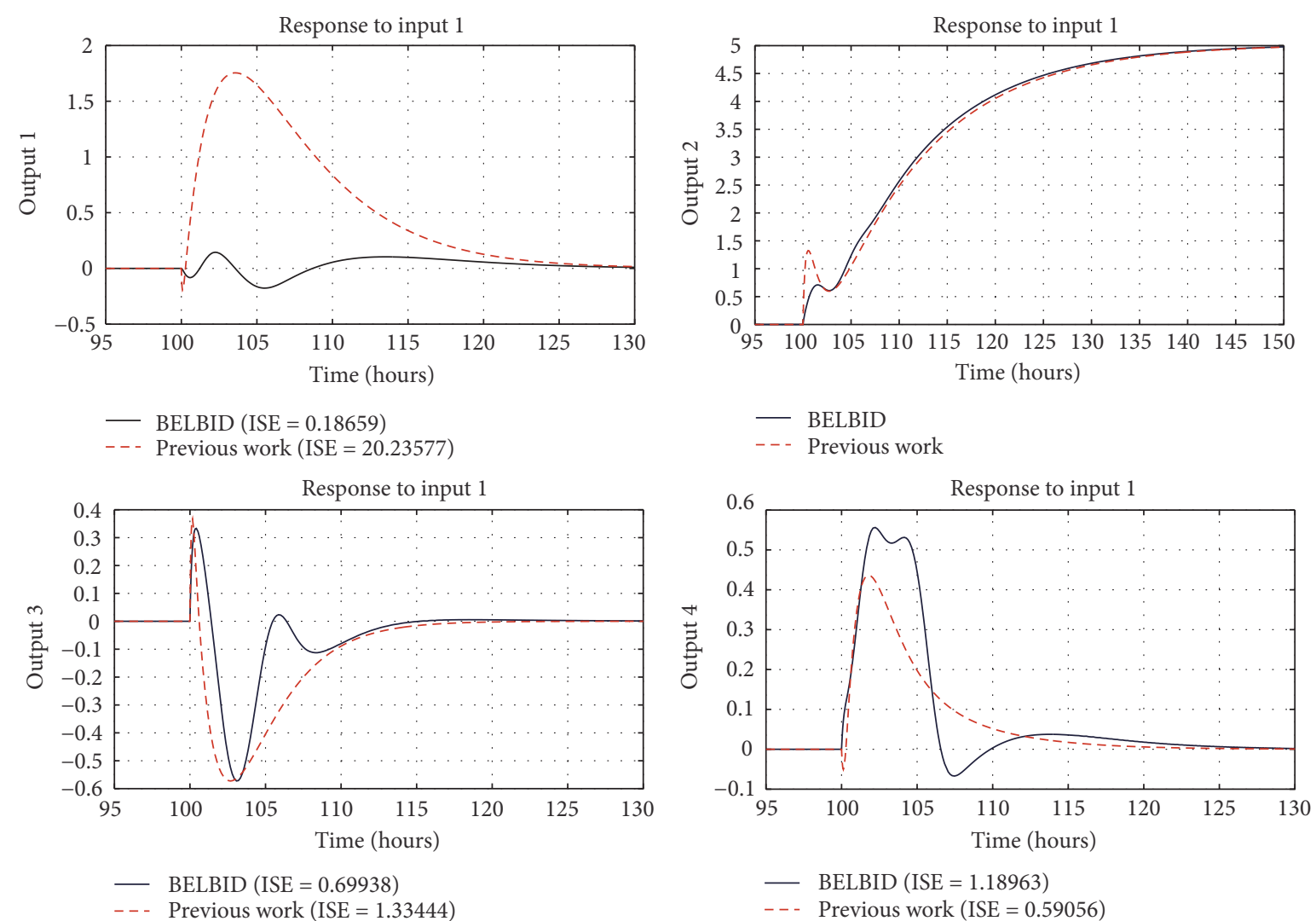

(a)
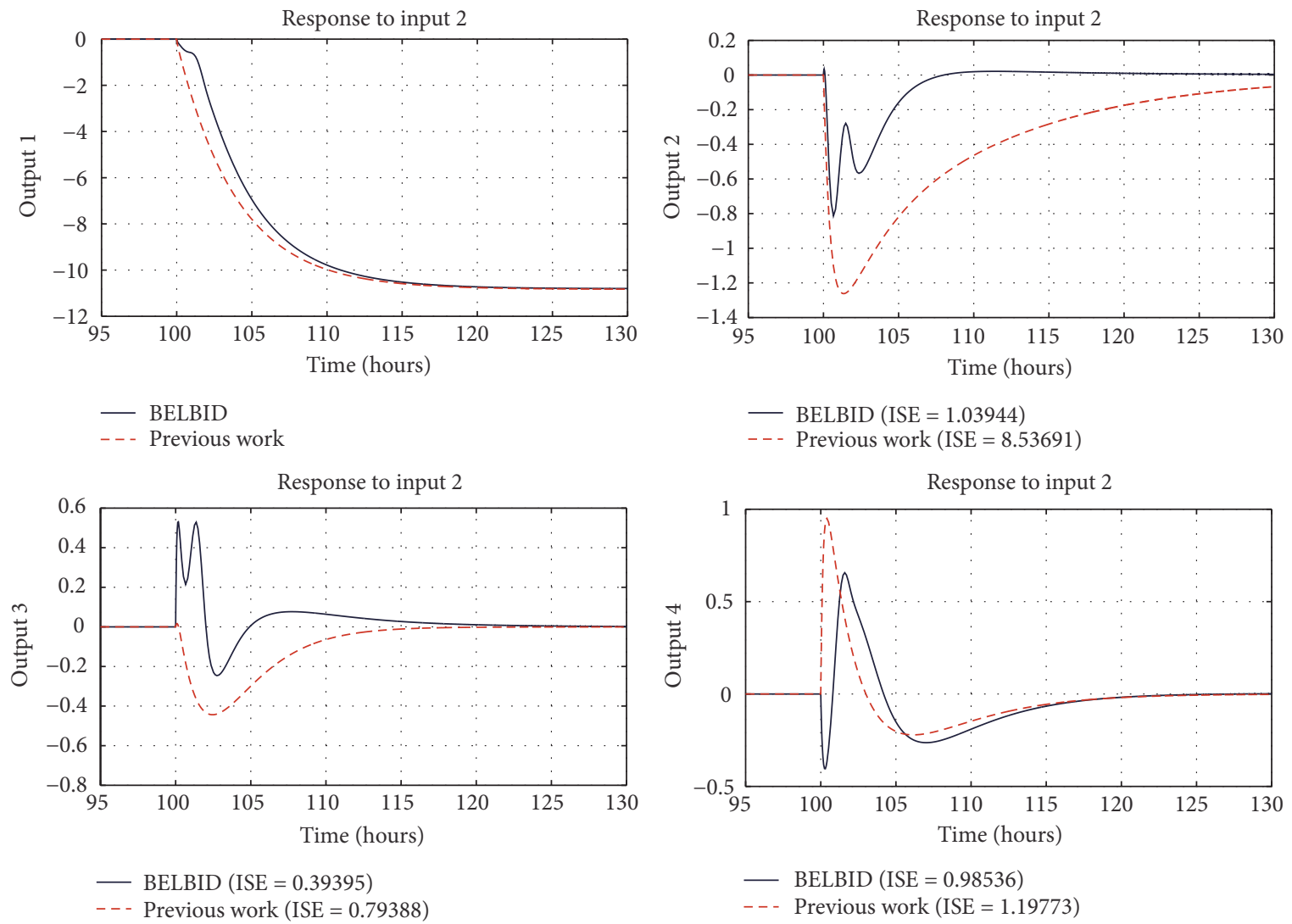

(b)

Figure 8: Continued. 

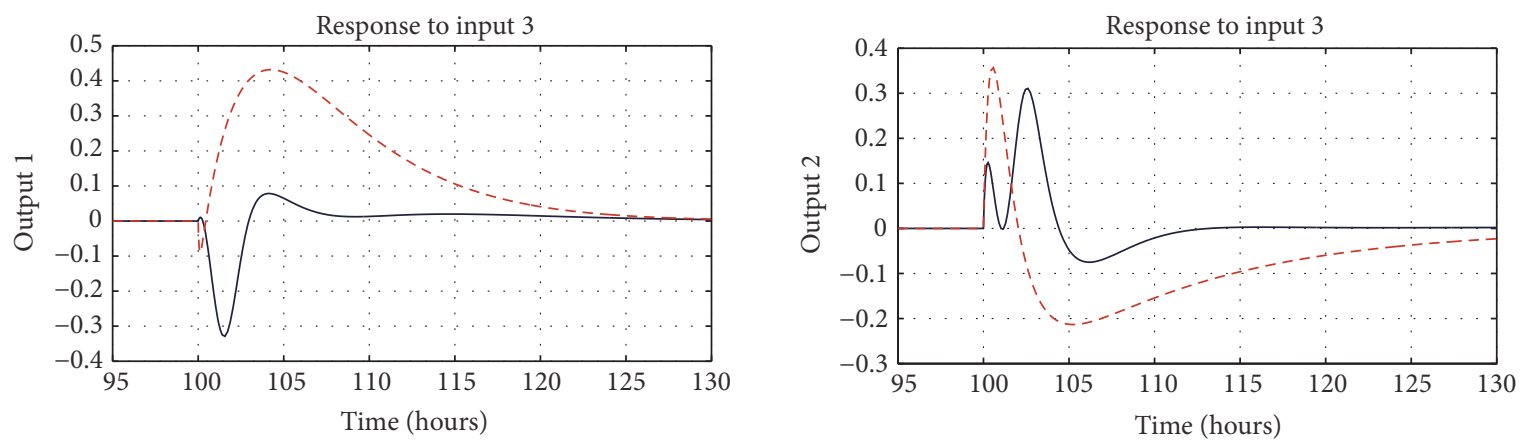

- BELBID (ISE $=0.14032$ )

- - - Previous work $($ ISE $=1.34556)$

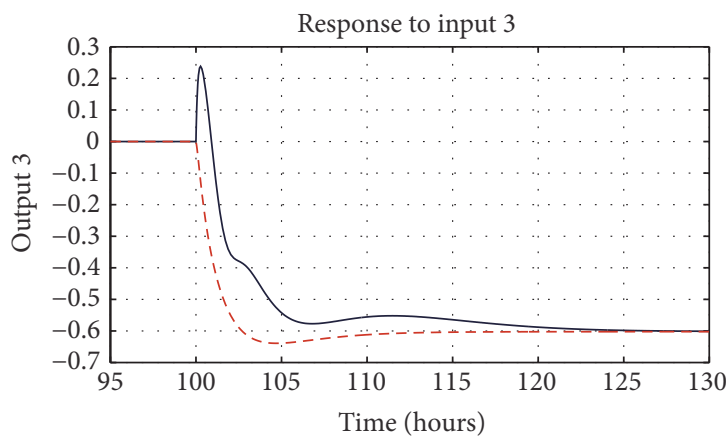

- BELBID (ISE $=0.14771$ )

- - - Previous work $($ ISE $=0.50096)$

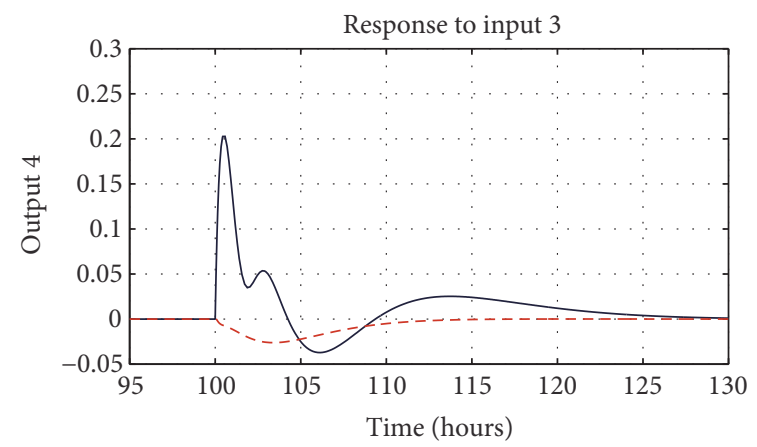

- BELBID

- - - Previous work

— BELBID $($ ISE $=0.04549)$

- $\ldots$ Previous work $($ ISE $=0.00310)$

(c)
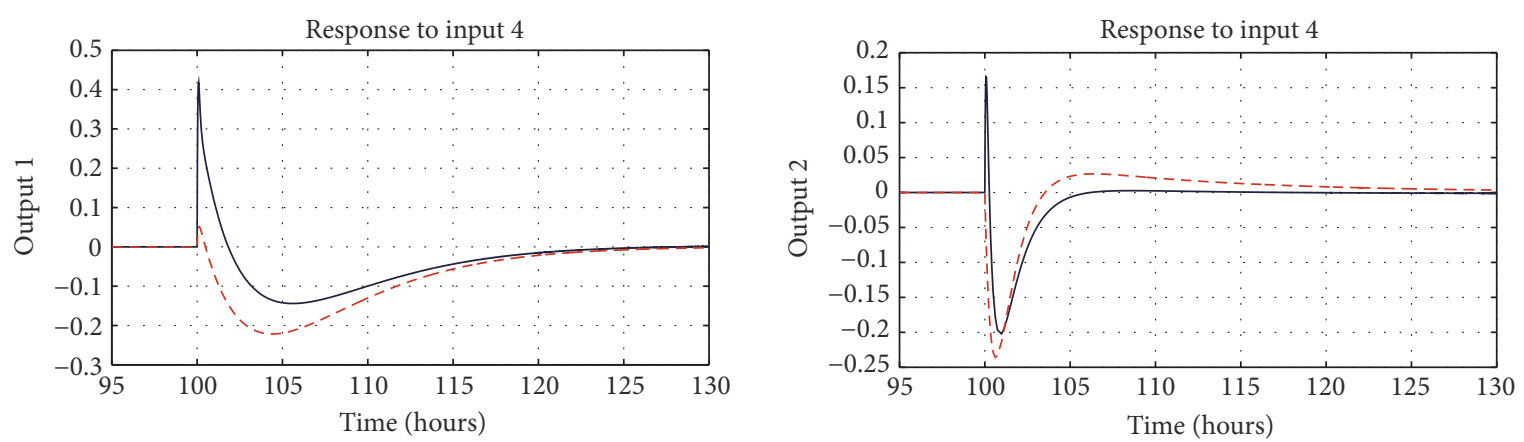

- BELBID (ISE $=0.21192$ )

- - - Previous work (ISE $=0.35868)$
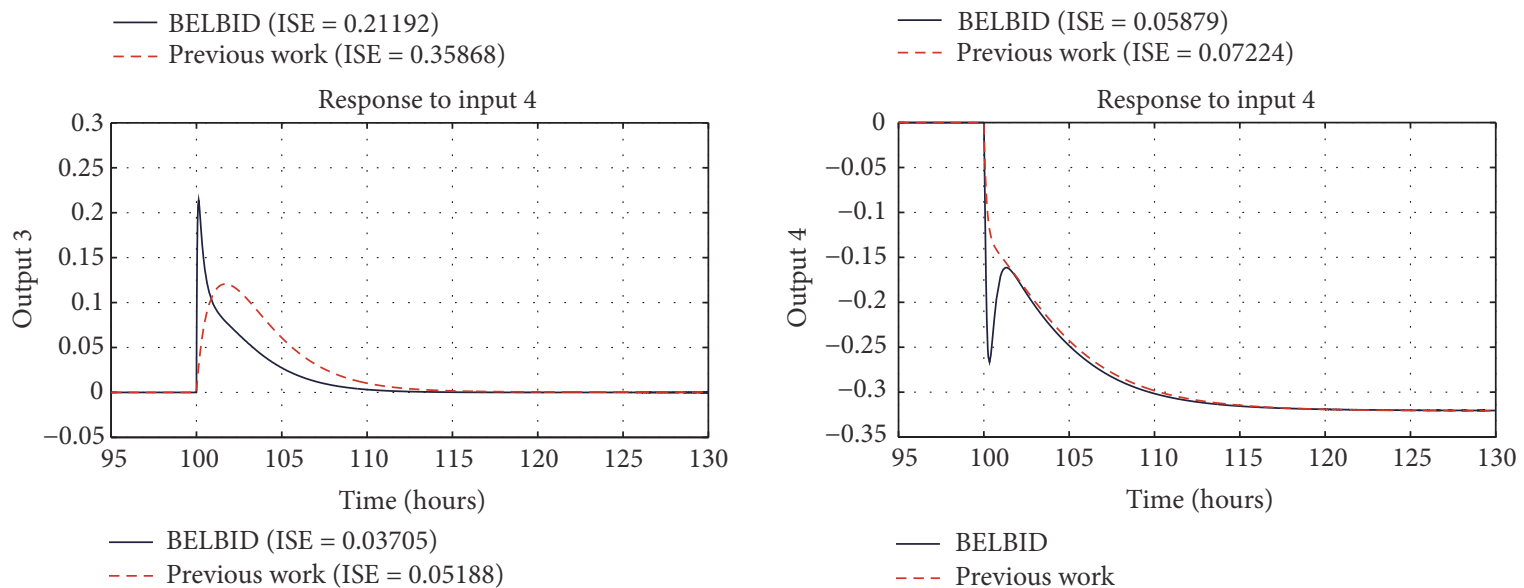

(d)

FIGURE 8: Detailed transient responses of the decoupled two-coupled distillation columns process via BELBID (solid line) and previous work (dashed line): (a) responses to step change in input 1, (b) responses to step change in input 2, (c) responses to step change in input 3 , and (d) responses to step change in input 4. 


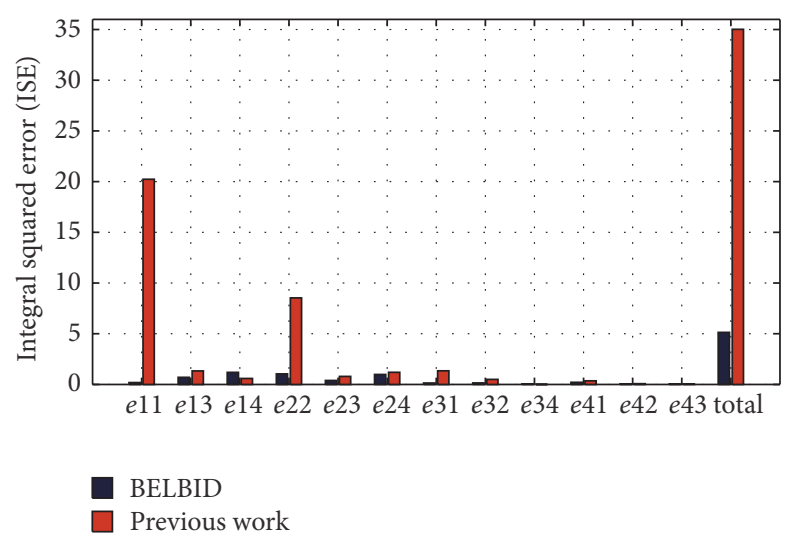

FIGURE 9: Bar graph of the coupling errors (ISE) of the BELBID and previous work.

emotions via the same inputs. This model is tailored to work as a MIMO decoupler of the two-coupled distillation columns. The decentralized approach of controlling MIMO systems, which include decoupling of the system and using set of SISO controllers, is preferable for many researches to deal with MIMO nonlinear systems. The gains' values and training rates of BELBID are optimized via PSO. In comparison with the PSO optimized compensation matrix, the BELBID remarkably decreases the coupling error due to the loops interactions. The total integral squared coupling error of the system is reduced by $85.3 \%$. The detailed responses declare that the BELBID decreases the total coupling error without degrading the responses of the paired outputs.

\section{Competing Interests}

The authors declare that there is no conflict of interests regarding the publication of this paper.

\section{References}

[1] I. Makaremi and B. Labibi, "Control of a distillation column: a decentralized approach," in Proceedings of the IEEE International Conference on Control Applications (CCA '06), pp. 711714, Munich, Germany, October 2006.

[2] A. M. El-Garhy and M. E. El-Shimy, "Development of decoupling scheme for high order MIMO process based on PSO technique," Applied Intelligence, vol. 26, no. 3, pp. 217-229, 2007.

[3] H. T. Dorrah, A. M. El-Garhy, and M. E. El-Shimy, "PSOBELBIC scheme for two-coupled distillation column process," Journal of Advanced Research, vol. 2, no. 1, pp. 73-83, 2011.

[4] Y. F. Hassan, "Rough sets for adapting wavelet neural networks as a new classifier system," Applied Intelligence, vol. 35, no. 2, pp. 260-268, 2011.

[5] M. Chen, S. S. Ge, and B. V. E. How, "Robust adaptive neural network control for a class of uncertain MIMO nonlinear systems with input nonlinearities," IEEE Transactions on Neural Networks, vol. 21, no. 5, pp. 796-812, 2010.

[6] A. M. El-Garhy, G. A. El-Sheikh, and M. H. El-Saify, "Fuzzy life-extending control of anti-lock braking system," Ain Shams Engineering Journal, vol. 4, no. 4, pp. 735-751, 2013.
[7] Y.-J. Liu, S. Tong, and C. L. P. Chen, "Adaptive fuzzy control via observer design for uncertain nonlinear systems with unmodeled dynamics," IEEE Transactions on Fuzzy Systems, vol. 21, no. 2, pp. 275-288, 2013.

[8] F. H. Pereira, W. A. L. Alves, L. Koleff, and S. I. Nabeta, "A twolevel genetic algorithm for large optimization problems," IEEE Transactions on Magnetics, vol. 50, no. 2, pp. 733-736, 2014.

[9] K. Shi and L. Li, "High performance genetic algorithm based text clustering using parts of speech and outlier elimination," Applied Intelligence, vol. 38, no. 4, pp. 511-519, 2013.

[10] C. Balkenius and J. Morén, "Emotional learning: a computational model of the amygdala," Cybernetics and Systems, vol. 32, no. 6, pp. 611-636, 2001.

[11] J. Moren, Emotion and Learning-A Computational Model of the Amygdala [Ph.D. thesis], Lund University, Lund, Sweden, 2002.

[12] C. Lucas, D. Shahmirzadi, and N. Sheikholeslami, "Introducing BELBIC: brain emotional learning based intelligent controller," Intelligent Automation and Soft Computing, vol. 10, no. 1, pp. 1122, 2004.

[13] S. Jafarzadeh, M. S. Fadali, and C. Lascu, "An emotional learning intelligent direct torque and flux controller design for induction motor," in Proceedings of the 4th Annual IEEE Energy Conversion Congress and Exposition (ECCE '12), pp. 3988-3992, Raleigh, NC, USA, September 2012.

[14] M. A. Rahman, M. A. Milasi, C. Lucas, B. N. Araabi, and T. S. Radwan, "Implementation of emotional controller for interior permanent-magnet synchronous motor drive," IEEE Transactions on Industry Applications, vol. 44, no. 5, pp. 1466-1476, 2008.

[15] G. Yang, Y. Cao, and L. Zhang, "Design of brain emotional learning model based hydraulic servo system," in Proceedings of the 2nd International Conference on Mechanic Automation and Control Engineering (MACE '11), pp. 4874-4876, chn, July 2011.

[16] M. Jafari, A. M. Shahri, and S. B. Shuraki, "Speed control of a digital servo system using brain emotional learning based intelligent controller," in Proceedings of the 4th Annual International Power Electronics, Drive Systems and Technologies Conference (PEDSTC '13), pp. 311-314, February 2013.

[17] M. A. Sharbafi, C. Lucas, and R. Daneshvar, "Motion control of omni-directional three-wheel robots by brain-emotionallearning-based intelligent controller," IEEE Transactions on Systems, Man and Cybernetics Part C: Applications and Reviews, vol. 40, no. 6, pp. 630-638, 2010.

[18] S. Jafarzadeh, R. Mirheidari, M. R. J. Motlagh, and M. Barkhordari, "Designing PID and BELBIC controllers in path tracking and collision problem in automated highway systems," in Proceedings of the 10th International Conference on Control, Automation, Robotics and Vision (ICARCV'08), pp. 1562-1566, Hanoi, Vietnam, December 2008.

[19] E. Bijami, R. Abshari, S. M. Saghaiannejad, and J. Askari, "Load frequency control of interconnected power system using brain emotional learning based intelligent controller," in Proceedings of the 19th Iranian Conference on Electrical Engineering (ICEE '11), IEEE, Tehran, Iran, May 2011.

[20] S. A. Aghaee, C. Lucas, and K. Amiri Zadeh, "Applying brain emotional learning based intelligent controller (BELBIC) to multiple-area power systems," Asian Journal of Control, vol. 14, no. 6, pp. 1580-1588, 2012.

[21] E. Lotfi and M. R. Akbarzadeh-T, "Emotional brain-inspired adaptive fuzzy decayed learning for online prediction problems," in Proceedings of the IEEE International Conference on Fuzzy Systems (FUZZ-IEEE '13), Hyderabad, India, July 2013. 
[22] H. Rouhani, M. Jalili, B. N. Araabi, W. Eppler, and C. Lucas, "Brain emotional learning based intelligent controller applied to neurofuzzy model of micro-heat exchanger," Expert Systems with Applications, vol. 32, no. 3, pp. 911-918, 2007.

[23] J. Kennedy and R. Eberhart, "Particle swarm optimization," in Proceedings of the IEEE International Conference on Neural Networks, pp. 1942-1948, Perth, Australia, December 1995.

[24] R. C. Eberhart, Y. Shi, and J. Kennedy, Swarm Intelligence (The Morgan Kaufmann Series in Evolutionary Computation), Morgan Kaufmann, San Francisco, Calif, USA, 1st edition, 2001.

[25] X. Liu and J. Qian, "Modeling, control, and optimization of ideal internal thermally coupled distillation columns," Chemical Engineering and Technology, vol. 23, no. 3, pp. 235-241, 2000.

[26] M. Miccio and B. Cosenza, "Control of a distillation column by type-2 and type-1 fuzzy logic PID controllers," Journal of Process Control, vol. 24, no. 5, pp. 475-484, 2014.

[27] S. Diaz, J. R. Perez-Correa, A. Cipriano, and M. FernandezFernandez, "Intelligent control applications on a binary distillation column," in Proceedings of the IEEE International Conference on Automatica (ICA-ACCA '16), pp. 1-8, IEEE, October 2016.

[28] Z. Chen, M. A. Henson, P. Belanger, and L. Megan, "Nonlinear model predictive control of high purity distillation columns for cryogenic air separation," IEEE Transactions on Control Systems Technology, vol. 18, no. 4, pp. 811-821, 2010.

[29] B. Yan, S. X.-D. Tan, L. Zhou, J. Chen, and R. Shen, "Decentralized and passive model order reduction of linear networks with massive ports," IEEE Transactions on Very Large Scale Integration (VLSI) Systems, vol. 20, no. 5, pp. 865-877, 2012.

[30] E. Kurniawan, Z. Cao, and Z. Man, "Design of decentralized repetitive control of linear MIMO system," in Proceedings of the IEEE 8th Conference on Industrial Electronics and Applications (ICIEA '13), pp. 427-432, Melbourne, Australia, June 2013.

[31] S. Kunimatsu, M. Ishitobi, and T. Fujii, "Decentralized PID control for systems with relative degree no more than 2," in Proceedings of the 51st Annual Conference on of the Society of Instrument and Control Engineers of Japan (SICE '12), pp. 11061109, Akita, Japan, August 2012.

[32] W. Lee, D. Yoon, J. Lee, S. Kim, and S. K. Park, "Decoupling matrix with rate one quasi-orthogonal STBC for eight transmit antennas," in Proceedings of the 2008 11th IEEE Singapore International Conference on Communication Systems (ICCS '08), pp. 73-76, November 2008.

[33] M. V. Pavan Kumar and N. Kaistha, "Decentralized control of a kinetically controlled ideal reactive distillation column," Chemical Engineering Science, vol. 63, no. 1, pp. 228-243, 2008.

[34] C. Rajapandiyan and M. Chidambaram, "Controller design for MIMO processes based on simple decoupled equivalent transfer functions and simplified decoupler," Industrial and Engineering Chemistry Research, vol. 51, no. 38, pp. 12398-12410, 2012.

[35] Z. Beheshti and S. Z. M. Hashim, "A review of emotional learning and it's utilization in control engineering," International Journal of Advances in Soft Computing and its Applications, vol. 2, no. 2, pp. 191-208, 2010.

[36] M. Jalili-Kharaajoo, "Nonlinear system identification using ANFIS based on emotional learning," in Advances in Artificial Intelligence-IBERAMIA 2004: 9th Ibero-American Conference on AI, Puebla, Mexico, November 22-26, 2004. Proceedings, vol. 3315 of Lecture Notes in Computer Science, pp. 697-707, Springer, Berlin, Germany, 2004.
[37] A. Gholipour, C. Lucas, and D. Shahmirzadi, "Purposeful prediction of space weather phenomena by simulated emotional learning," International Journal of Modelling and Simulation, vol. 24, no. 2, pp. 65-72, 2004.

[38] M. Parsapoor and U. Bilstrup, "Brain Emotional Learning Based Fuzzy Inference System (BELFIS) for solar activity forecasting," in Proceedings of the IEEE 24th International Conference on Tools with Artificial Intelligence (ICTAI '12), pp. 532-539, Athens, Greece, November 2012.

[39] J. Abdi, B. Moshiri, B. Abdulhai, and A. K. Sedigh, "Forecasting of short-term traffic-flow based on improved neurofuzzy models via emotional temporal difference learning algorithm," Engineering Applications of Artificial Intelligence, vol. 25, no. 5, pp. 1022-1042, 2012.

[40] E. Lotfi, S. Setayeshi, and S. Taimory, "A neural basis computational model of emotional brain for online visual object recognition," Applied Artificial Intelligence, vol. 28, no. 8, pp. 814-834, 2014.

[41] N. Abdelkarim, A. E. Mohamed, A. M. El-Garhy, and H. T. Dorrah, "A New Hybrid BFOA-PSO optimization technique for decoupling and robust control of two-coupled distillation column process," Computational Intelligence and Neuroscience, vol. 2016, Article ID 8985425, 17 pages, 2016. 


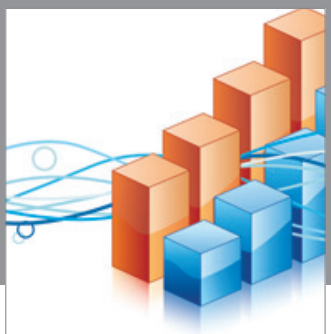

Advances in

Operations Research

vatem alat4

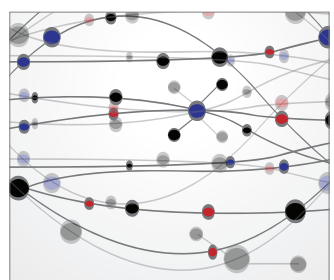

\section{The Scientific} World Journal
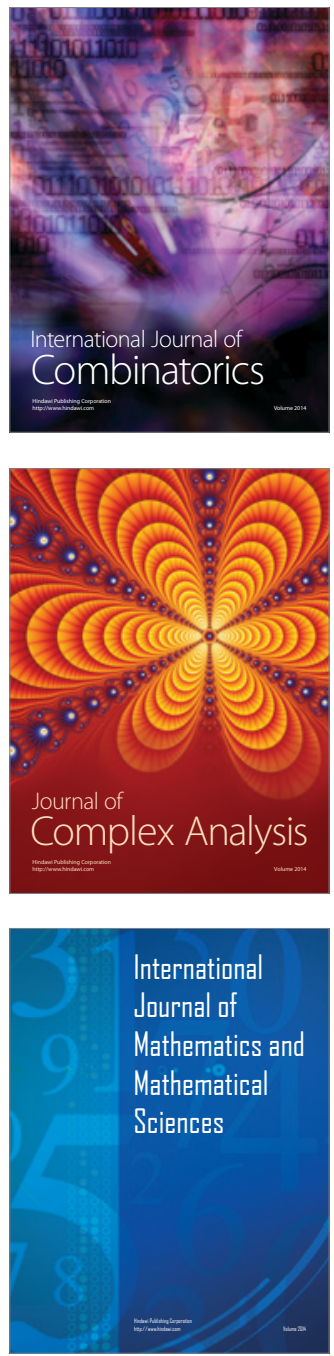
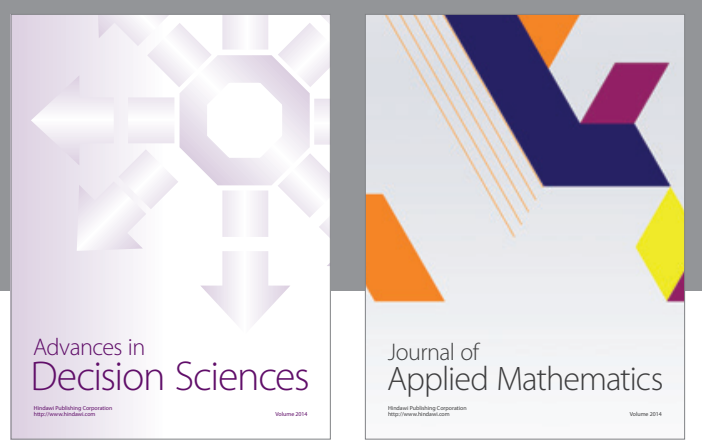

Algebra

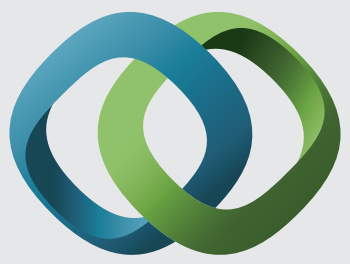

\section{Hindawi}

Submit your manuscripts at

https://www.hindawi.com
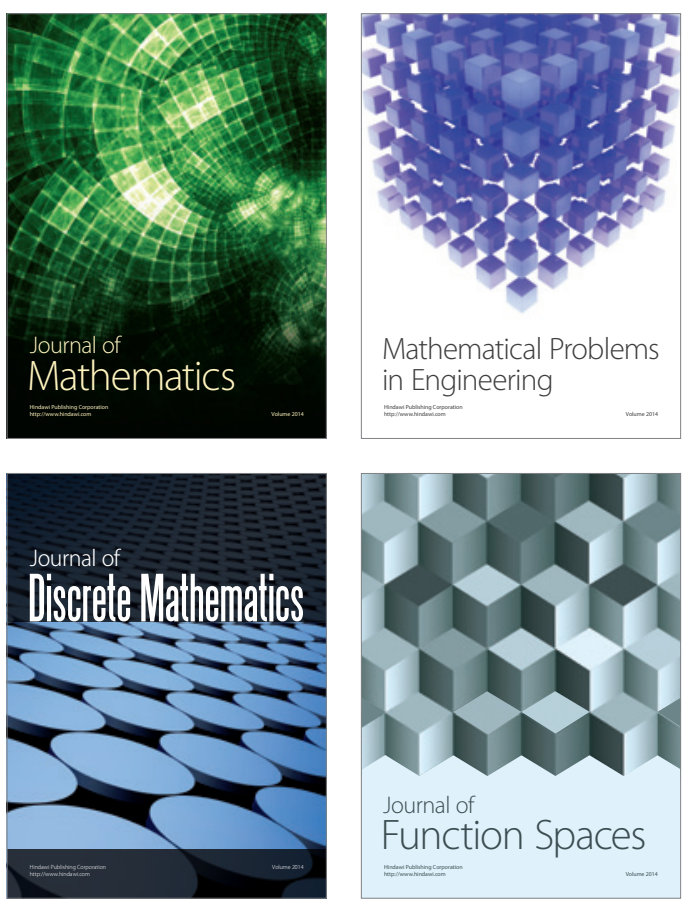

Mathematical Problems in Engineering
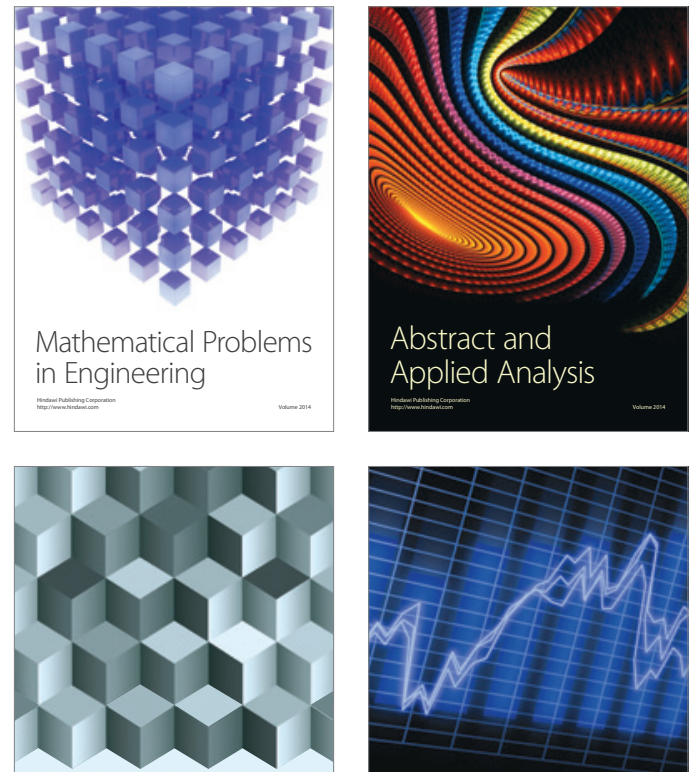

Journal of

Function Spaces

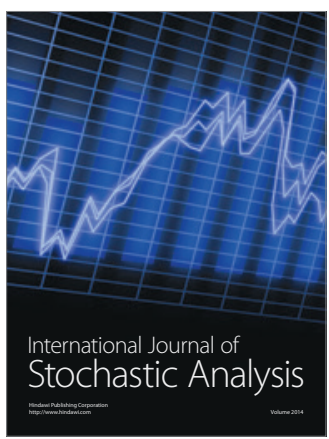

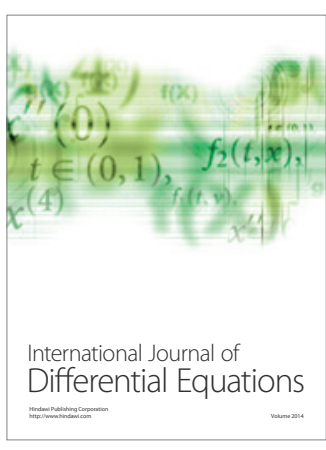
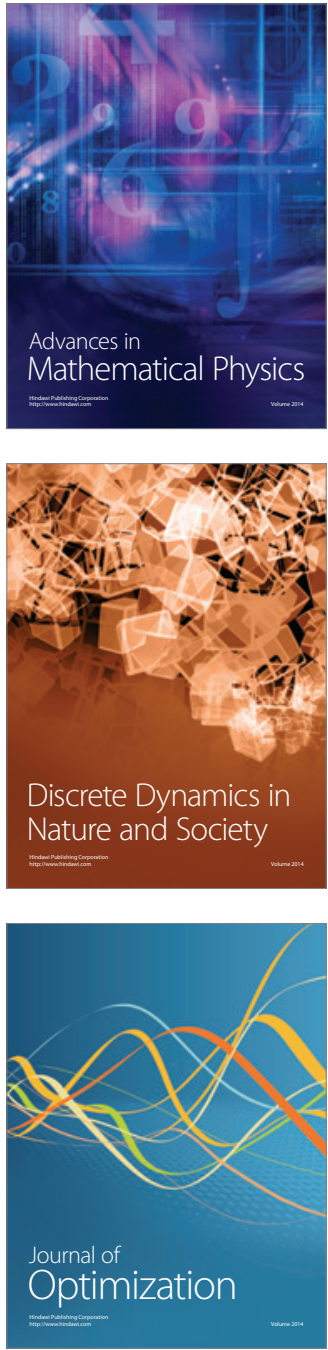